\title{
Erratum to: Fibers of tropicalization
}

\author{
Sam Payne
}

Published online: 16 October 2012

(C) Springer-Verlag Berlin Heidelberg 2012

\section{Erratum to: Math. Z. (2009) 262:301-311 DOI 10.1007/s00209-008-0374-x}

Abstract This note explains an error in Proposition 5.1 of "Fibers of tropicalization", Math. Z. 262 (2009), no. 2, 301-311, discovered by W. Buczynska and F. Sottile, and fills the resulting gap in the proof of the paper's main theorem.

Part (3) of Proposition 5.1 in [8] claims that if $X$ is a subvariety of a torus $T$ containing the identity then there is a split surjection $\varphi: T \rightarrow T^{\prime}$ such that the image of $X$ is a hypersurface and the intersection of the initial degeneration $X_{0}$ with the kernel of $\varphi_{0}$ is $\left\{1_{T}\right\}$. This claim is false, and the following is a counterexample.

Example 1 Suppose the characteristic of $K$ is not 2, and let $X$ be a curve in a threedimensional torus containing all eight 2-torsion points of $T$. Then $X_{0}$ contains all eight 2-torsion points of $T_{0}$ and, for any projection $\varphi$ from $T$ to a two dimensional torus, the kernel of $\varphi_{0}$ contains four 2-torsion points, all of which are in $X_{0}$.

The falsehood of part (3) of Proposition 5.1 leaves an essential gap in the proof of Theorem 4.1, which is the main result of [8]. This result has now been proved independently by different means, including nonarchimedean analysis [4, Proposition 4.14] and noetherian approximation [7, Theorem 4.2.5]. The original proposed method of proof using split surjections of tori to decrease the codimension may be of independent interest, but the error in Proposition 5.1 interferes with the reduction to the hypersurface case.

Here, we complete the proof of Theorem 4.1 via the original method of split surjections of tori by projecting even further, onto a torus of dimension equal to $\operatorname{dim} X$, and using the going-down theorem for finite extensions of an integrally closed domain.

The online version of the original article can be found under doi:10.1007/s00209-008-0374-x.

S. Payne $(\varangle)$

Department of Mathematics, Yale University,

10 Hillhouse Ave, New Haven, CT 06511, USA

e-mail: sam.payne@yale.edu 
Remark 2 The second main result of [8] is Corollary 4.2, which says that the fibers of the classical tropicalization map from $X(K)$ to $N_{G}$ are Zariski dense. The error in Proposition 5.1 does not create a serious gap in the proof of this weaker result; the original arguments can be modified, as follows, to obtain Corollary 4.2 without deducing it from Theorem 4.1. Parts (1) and (2) of Proposition 5.1 reduce Corollary 4.2 to the hypersurface case, and Proposition 6.1 shows that if $X$ is a hypersurface then $\operatorname{Trop}^{-1}(v) \cap X(K)$ is infinite. The argument in Section 6.3 then goes through with $\underline{x}$ and $\mathfrak{T r o p}^{-1}(\underline{x})$ replaced by $v$ and $\operatorname{Trop}^{-1}(v)$, respectively.

To prove Theorem 4.1, we first consider the special case when $X$ is a torus.

Lemma 3 For any $v \in N_{G}$ and $\underline{x} \in T_{v}(k)$, the fiber $\mathfrak{T r o p}^{-1}(\underline{x})$ is Zariski dense in $T$.

Proof After translation, we may assume that $v=0$ and $\underline{x}=1_{T}$. If $T=K^{*}$ is onedimensional then $\mathfrak{T r o p}^{-1}\left(1_{T}\right)$ is identified with the subset $1+\mathfrak{m}$ of the valuation ring $R$, which is infinite and hence Zariski dense. For $T$ of arbitrary dimension $n$, choose an isomorphism from $T$ to $\left(K^{*}\right)^{n}$. Then Trop ${ }^{-1}\left(1_{T}\right)$ is identified with the product of dense sets $(1+\mathfrak{m})^{n}$, and is therefore dense.

Next, we choose a suitable projection from $X$ to a torus of dimension equal to $\operatorname{dim} X$. Say $X$ has dimension $d$. Recall that the set of $v$ in $N_{\mathbb{R}}$ such that $X_{v}$ is nonempty is the underlying set of a finite polyhedral complex $\Delta$ of pure dimension $d$. Projecting along a general rational subspace of codimension $d$ in $N_{\mathbb{Q}}$ maps each face of $\Delta$ isomorphically onto its image. Such a projection corresponds to a split surjection of tori $\varphi: T \rightarrow T^{\prime}$ with the property that, for each $v^{\prime} \in N_{G}^{\prime}$, the preimage

$$
\phi^{-1}\left(v^{\prime}\right) \cap \operatorname{Trop}(X)
$$

is finite, where $\phi: N_{G} \rightarrow N_{G}^{\prime}$ is the linear map induced by $\varphi$.

Say $v_{0}, \ldots, v_{s}$ are the finitely many preimages in $\Delta$ of $\phi(v)$, where $v_{0}=v$. Each of the schemes $T_{v_{0}}, \ldots, T_{v_{s}}$ over $\operatorname{Spec} R$ contains the torus $T_{K}$ as an open subscheme, and the morphisms $\varphi_{v_{i}}: T_{v_{i}} \rightarrow T_{\phi(v)}^{\prime}$ all agree on $T_{K}$. Therefore, we can glue these schemes and morphisms to get

$$
T_{v_{0}} \cup \cdots \cup T_{v_{s}} \rightarrow T_{\phi(v)}^{\prime}
$$

over $\operatorname{Spec} R$. We write $\Phi$ for the restriction of this morphism to the closure of $X$ and prove Theorem 4.1 using the following technical result.

Proposition 4 The morphism $\Phi: \mathcal{X}_{v_{0}} \cup \cdots \cup \mathcal{X}_{v_{s}} \rightarrow T_{\phi(v)}^{\prime}$ is finite.

Here $\mathcal{X}_{v_{i}}$ denotes the closure of $X$ in $T_{v_{i}}$. The schemes $\mathcal{X}_{v_{i}}$ and $T_{\phi(v)}^{\prime}$ over $\operatorname{Spec} R$ are not noetherian, but this does not create any additional difficulties. We deduce Theorem 4.1 from Proposition 4 using the going-down theorem for finite extensions of an integrally closed domain, which has no noetherian hypothesis.

Proof of Theorem 4.1 First, we show that $R\left[M^{\prime}\right]^{\phi(v)}$ is an integrally closed domain. Translating by a point in $\operatorname{Trop}^{-1}(\phi(v))$ induces an isomorphism from $R\left[M^{\prime}\right]^{\phi(v)}$ to $R\left[M^{\prime}\right]$ which is a localization of a polynomial ring over the integrally closed domain $R$, and hence an integrally closed domain, by [5, Proposition 17.B(2)] and [6, Example 9.3].

By Lemma 3, the points $x^{\prime}$ in $\mathfrak{T r o p}^{-1}\left(\varphi_{v}(\underline{x})\right)$ are dense in $T^{\prime}$. By the going-down theorem for finite extensions of an integrally closed domain [6, Theorem 9.4(ii)], for each such $x^{\prime}$ there is a point $x \in X(K)$ specializing to $\underline{x}$ such that $\varphi(x)=x^{\prime}$. This shows that the image of $\mathfrak{T r o p}^{-1}(\underline{x}) \cap X(K)$ is Zariski dense in $T^{\prime}$. Since $\varphi$ is finite, it follows that $\mathfrak{T r o p}^{-1}(\underline{x}) \cap X(K)$ is Zariski dense in $X$, as required. 
It remains to prove Proposition 4. To do this, we work with $G$-admissible fans and the associated toric schemes over $\operatorname{Spec} R$, as in [4], to which we refer the reader for details of these constructions. Choose a $G$-admissible fan structure $\Sigma^{\prime}$ on $N_{\mathbb{R}}^{\prime} \times \mathbb{R}_{\geq 0}$ that contains $\mathbb{R}_{\geq 0} \cdot(\phi(v), 1)$ as a 1-dimensional face. Let $\Sigma$ be a $G$-admissible fan on $N_{\mathbb{R}} \times \mathbb{R}_{\geq 0}$ such that

(1) For each face $\tau \in \Delta$, the cone $\mathbb{R}_{\geq 0} \cdot(\tau \times 1)$ is a union of faces of $\Sigma$.

(2) For each face $\sigma \in \Sigma$, the image $(\phi \times 1)(\sigma)$ is contained in a face of $\Sigma^{\prime}$.

Let $\mathcal{Y}_{\Sigma}$ and $\mathcal{Y}_{\Sigma^{\prime}}$ be the corresponding toric schemes over $\operatorname{Spec} R$, as defined in [4, Section 7], containing $T_{K}$ and $T_{K}^{\prime}$ as dense open subschemes, respectively, and let $\mathcal{X}_{\Sigma}$ be the closure of $X$ in $\mathcal{Y}_{\Sigma}$. Since $\Sigma^{\prime}$ contains $\mathbb{R}_{\geq 0} \cdot(\phi(v), 1)$, the toric scheme $\mathcal{Y}_{\Sigma^{\prime}}$ contains $T_{\phi(v)}^{\prime}$ as an open subscheme. Then, by (1) and (2), the cones $\mathbb{R}_{\geq 0}\left(v_{i}, 1\right)$ must be cones in $\Sigma$, for $i=0, \ldots, s$, and hence $\mathcal{X}_{\Sigma}$ contains $\mathcal{X}_{v_{0}} \cup \cdots \cup \mathcal{X}_{v_{s}}$ as an open subscheme as well.

The morphism $\varphi: T_{K} \rightarrow T_{K}^{\prime}$ extends to a morphism of toric schemes from $\mathcal{Y}_{\Sigma}$ to $\mathcal{Y}_{\Sigma^{\prime}}$, by [4, 11.9], and we write $\bar{\Phi}$ for the restriction of this morphism to $\mathcal{X}_{\Sigma}$. The morphism $\Phi$ in Proposition 4 is the restriction of $\bar{\Phi}$ to $\mathcal{X}_{v_{0}} \cup \cdots \cup \mathcal{X}_{v_{s}}$.

Lemma 5 The morphism $\bar{\Phi}: \mathcal{X}_{\Sigma} \rightarrow \mathcal{Y}_{\Sigma^{\prime}}$ is proper and of finite presentation.

Proof By [4, Proposition 11.12] and (1), the scheme $\mathcal{X}_{\Sigma}$ is proper over $\operatorname{Spec} R$. Since $\mathcal{Y}_{\Sigma^{\prime}}$ is separated over $\operatorname{Spec} R$ [4, Lemma 7.8], it follows that $\mathcal{X}_{\Sigma}$ is proper over $\mathcal{Y}_{\Sigma^{\prime}}$ [1, Corollary 5.4.3(i)].

Since $\Sigma$ is $G$-admissible and $G$ is divisible, the toric scheme $\mathcal{Y}_{\Sigma}$ is of finite presentation over $\operatorname{Spec} R$ [4, Proposition 6.7], and in particular it is of finite type over $\operatorname{Spec} R$. Therefore, $\mathcal{X}_{\Sigma}$ is of finite type over $\operatorname{Spec} R$. Since $\mathcal{X}_{\Sigma}$ is the closure of its generic fiber, by construction, it is also flat, and hence of finite presentation over $\operatorname{Spec} R$ [9, Corollary 3.4.7]. Hence $\mathcal{X}_{\Sigma}$ is also of finite presentation over $\mathcal{Y}_{\Sigma}[2$, Proposition 1.6.2(v)].

Proof of Proposition 4 By [4, Lemma 11.6], the union $\mathcal{X}_{v_{0}} \cup \cdots \cup \mathcal{X}_{v_{s}}$ is the full preimage of $T_{\phi(v)}^{\prime}$ under $\Phi$. Therefore, by Lemma $5, \Phi$ is proper and of finite presentation. To show that $\Phi$ is finite, it remains to show that it has finite fibers [3, Theorem 8.11.1].

We begin with the general fiber of $T_{\phi(v)}^{\prime}$. If $x^{\prime} \in T^{\prime}(K)$, then the tropicalization of the fiber $\Phi^{-1}\left(x^{\prime}\right)$ must be contained in $\phi^{-1}\left(\operatorname{Trop}\left(x^{\prime}\right)\right) \cap \operatorname{Trop}(X)$, which is finite by construction. Therefore, the fiber must be zero-dimensional and hence finite, since $\Phi$ is of finite presentation.

It remains to check the special fiber of $T_{\phi(v)}^{\prime}$. Suppose $\underline{x}^{\prime}$ is in $T_{\phi(v)}^{\prime}(k)$. The preimage of $T_{\phi_{v}}^{\prime}(k)$ is the disjoint union $X_{v_{0}} \cup \cdots \cup X_{v_{s}}$. Therefore, the tropicalization of $\Phi^{-1}\left(\underline{x}^{\prime}\right) \cap X_{v_{i}}$ must be contained in the fiber over 0 under the natural map from the star of $v_{i}$ in $\operatorname{Trop}(X)$ to $N_{\mathbb{R}}^{\prime}$, which is, again, finite by construction. It follows that the fiber over $\underline{x}^{\prime}$ must be zerodimensional, and hence finite, as required.

Remark 6 In addition to the error in Proposition 5.1, there is an unrelated sign error in Section 3 of [8], which appears in four places. At the bottom of p. 305, the tilted group ring should be defined as

$$
R[M]^{v}=\bigoplus_{u \in M} \mathfrak{m}^{-\langle u, v\rangle} .
$$

The quotient $k\left[T_{v}\right]$ of this ring by the ideal generated by $\mathfrak{m}$ is then $\bigoplus_{u \in M} k^{-\langle u, v\rangle}$, the weight function on monomials is given by $b x^{u} \mapsto v(b)+\langle u, v\rangle$, and the weight of the monomial $a_{u, i} x^{u} t^{i}$ in Example 3.3 is $i+\langle u, v\rangle$. 
Acknowledgments I am most grateful to W. Buczynska and F. Sottile for their careful reading and for bringing these mistakes to my attention. This work was supported in part by NSF DMS 1068689 and completed during a visit to the Max Planck Institute for Mathematics in Bonn, Germany.

\section{References}

1. Grothendieck, A.: Éléments de géométrie algébrique. II. Étude globale élémentaire de quelques classes de morphismes. Inst. Hautes Études Sci. Publ. Math. 8, 222 (1961)

2. Grothendieck, A.: Éléments de géométrie algébrique. IV. Étude locale des schémas et des morphismes de schémas. I. Inst. Hautes Études Sci. Publ. Math. 20, 259 (1964)

3. Grothendieck, A.: Éléments de géométrie algébrique. IV. Étude locale des schémas et des morphismes de schémas. III. Inst. Hautes Études Sci. Publ. Math. 28, 255 (1966)

4. Gubler, W.: A guide to tropicalizations, preprint, arXiv:1108.6126v2, 2012

5. Matsumura, H.: Commutative algebra, second ed., Mathematics Lecture Note Series, vol. 56, Benjamin/Cummings Publishing Co., Inc., Reading (1980)

6. Matsumura, H.: Commutative ring theory, second ed. Cambridge Studies in Advanced Mathematics, vol. 8. Cambridge University Press, Cambridge (1989)

7. Osserman, B., Payne, S.: Lifting tropical intersections. preprint, arXiv:1007.1314v1 (2010)

8. Payne, S.: Fibers of tropicalization. Math. Z. 262(2), 301-311 (2009)

9. Raynaud, M., Gruson, L.: Critères de platitude et de projectivité. Techniques de "platification" d'un module. Invent. Math. 13, 1-89 (1971) 